\title{
L'epistola del Ventoso e le misure della rappresentazione petrarchesca della realtà
}

\author{
Francesco Paolo Botti \\ Università Federico II Napoli
}

\begin{abstract}
Nell'epistola del Ventoso Petrarca esprime non solo la prospettiva «agostiniana» di una nuova concezione, antinaturalistica etica introspettiva, del sapere ma mette in scena il superamento della rappresentazione teologico-medievale del mondo (il simbolismo dell'alto viene sottratto al suo significato di purificazione e di innalzamento al divino, in un primo momento esplicitamente richiamato, per essere collegato alla negatività della curiositas scientifica) e introduce potentemente nella visione delle cose la dimensione della temporalità.
\end{abstract}

Parole chiave: Petrarca, Epistola del Ventoso, spazio letterario, paesaggio.

\section{Abstract}

The epistle of Ventoso Petrarca expresses the «Augustinian» prospective of a new conception of understanding, introspective ethical anti-naturalism. However, it also creates the overpowering of the theological-medieval representation of the world (subtracting high symbolism from its meaning of purification and ascension to the divine, in an initial, clearly-defined moment, through its relation to the negativity of scientific curiositas) and strongly introduces the dimension of time into the grand scheme of things.

Key words: Petrarch, The Ventoso Epistle, literary space, lansdcape.

Nell'epistola del monte Ventoso ${ }^{1}$ la «filosofia» petrarchesca prende forma di racconto, un racconto autobiografico che propone la concezione agostiniana della verità interiore come traguardo di una vicenda di alternative e lacerazioni morali che affliggono l'io narrante. L'appello del protoumanista al rinnovamento, alla rifondazione, anzi, del sapere si sostanzia del pathos di una storia individuale, la propria, dell'intimità rovente di un'anima e del suo segreto con-

1. La familiare IV 1 sarà citata con la sola indicazione dei paragrafi e secondo il testo stabilito da V. Rossi, in F. Petrarca, Le Familiari (a cura di Vittorio Rossi), vol. I, Firenze: Sansoni, 1933, p. 153-61. 
flitto; e l'enunciato intellettuale si «incarna» nelle figurazioni e nei simboli della letteratura, nell' "eloquenza» con cui essa sa rendere umanamente partecipabili le avventure del pensiero. Ma allora, in quanto testo letterario, l'epistola non esaurisce con l'esplicitazione del proprio messaggio filosofico, dedotto dalle Confessioni di Agostino, un campo di significati che soltanto un'analisi attenta della tessitura compositiva può restituire nella ricchezza, complessità e anche contraddittorietà delle sue tensioni. Qui, ben al di là dell'accensione emotiva che, secondo Petrarca, l'incanto retorico dei grandi moralisti latini imprime ai contenuti dottrinali, il modo nuovo di guardare la realtà che lo scrittore va instaurando è del tutto immanente alla forma stessa della rappresentazione, al gioco della sua dinamica narrativa e dei suoi effetti figurali.

Se la lettera è il resoconto di un preciso evento biografico, l'escursione sul Ventoux, che la cadenza marcatamente allusiva dell'affabulazione spinge, però, alla più impegnativa amplificazione simbolica, e pertanto il cammino verso la vetta assurge a simbolo di tutta una vita, dell'itinerario esistenziale dell'autore fra abbandono alle vanità mondane e proponimenti penitenziali, la prima evidenza che si impone all'attenzione critica riguarda, per l'appunto, la parabola discontinua, irregolare fino all'incongruenza descritta da questo processo di simbolizzazione.

Petrarca, dunque, trasfigura l'occasione autobiografica della gita in montagna riplasmandola, per esaltarne al massimo grado le potenzialità significative, in chiave scopertamente metaforica: nella chiave della vetusta, elementare, e nelle sue opere pervasiva, metafora del cammino della vita, il «cammin di nostra vita». ${ }^{2}$ E la vita è un cammino lungo il quale prima o poi si presenta al viandante una biforcazione che lo obbliga a decidere la strada da prendere, l'indirizzo da dare alla propria esistenza, come l'Ercole al bivio del famoso apologo classico (che, conosciuto attraverso le pagine del De officiis ciceroniano, Petrarca

2. Che, peraltro, nel quadro della mentalità medievale è assai meno un tropo — più o meno sbiadito - che un'autentica modalità conoscitiva ed etica, come, a proposito dell' incipit della Commedia, sottolineava con vigore Salvatore BATTAGLIA: "Che la vita umana sia considerata come un cammino, è, si può dire, un luogo comune, è una metafora usuale e tradizionale, che è possibile ritrovare anche nella letteratura classica, oltre che in quella medievale. Ma non tutti i cammini hanno lo stesso tracciato, e non tutte le strade portano alla stessa meta. Intendere l'esistenza umana come un viaggio significava per il Medioevo definirla nel suo valore più intrinseco, più spirituale. Per la religiosità medievale la vita era più esattamente una peregrinazione. La condizione morale dell'uomo cristiano era [...] quella di chi si sente in esilio, come confinato su questa terra, mentre continua a portare nella propria interiorità l'immagine della patria lontana, intuita ma non conosciuta, viva e reale nella coscienza ma ogni giorno più distante $\mathrm{e}$ remota. E' un pellegrinaggio dell'anima che agogna di rivedere la regione da dove si è temporaneamente separata. E questa configurazione interiore della vita non era soltanto un'idea, una similitudine, un'allegoria, come noi moderni siamo soliti ritenere; bensì era uno stato morale, una situazione dello spirito oltre che dell'intelletto. Il nostro fraintendimento, e diciamo equivoco, è di ritenere che il verso di Dante raffiguri un'immagine, un'allegoria: ed è invece una proiezione reale, è una cifra della sua fede, sua e dei suoi contemporanei. Cioè, Dante non intende istituire nel primo verso una similitudine (la vita umana paragonata a un cammino), ma vuole definire la nostra esistenza nella sua fondamentale condizione. Che è appunto un viaggio, un cammino, un pellegrinaggio dell'anima esiliata dalla sua vera patria» in Esemplarità e antagonismo nel pensiero di Dante [Parte prima], Napoli: Liguori, 1975, p. 62-3. 
riprende nel De vita solitaria). ${ }^{3}$ D'altronde, ricorre più volte nel Canzoniere «il motivo topico [...] del "bivio pitagorico": la via di sinistra, agevole, conduce a perdizione, quella di destra, malagevole, porta alla salvezza»; ${ }^{4}$ e, appunto, «è in gran parte giocata su una variazione di questo motivo la lettera che racconta dell'ascensione al Ventoso». ${ }^{5}$ Ma qui la difficoltà della via, e della prova, è legata alla fatica del salire. Il simbolismo della narrazione si adempie sostanzialmente nella dimensione verticale della spazialità; si sa, infatti, che «nel quadro di orientamento dello spazio simbolico, là dove l'antichità greco-romana aveva attribuito un ruolo preminente alla contrapposizione destra-sinistra, il cristianesimo, pur continuando a conferire un importante valore a tale coppia antinomica, del resto presente nell'Antico e nel Nuovo Testamento, aveva ben presto privilegiato il sistema alto-basso, destinato nel Medioevo a orientare, attraverso la spazializzazione del pensiero, la dialettica essenziale dei valori cristiani». ${ }^{6}$ Quella dell'ascendere il monte è una figura troppo aperta e trasparente nei suoi significati di elevazione spirituale, di innalzamento al divino, di perfezionamento interiore, perché valga la pena dilungarvisi più di tanto. L'archetipo del

3. "Ipse Hercules in solitudine sanum illud consilium vite cepit, cuius libro priore mentionem feci, quando velut in bivio diu multumque hesitans, ad postremum spreta voluptatis via semitam virtutis arripuit» (F. Petrarca, De vita solitaria, in Prose [a cura di G. Martellotti e di P.G. Ricci, E. Carrara, E. Bianchi], Milano-Napoli: Ricciardi, 1955, p. 550; il luogo precedente a cui accenna è a p. 332). Osserva Nicholas MANn (Pétrarque: Les voyages de l'esprit, Grenoble: Millon, 2004, p. 10): «Il semble d'ailleurs que Pétrarque soit le premier écrivain depuis près de mille ans à faire revivre cette histoire, à laquelle il donne une nouvelle orientation en intégrant dans le récit de Cicéron l'image du croisement avec toutes ses résonances pythagoriciennes».

4. M. Santagata, nota ai v. 120-1 di RVF 264, in F. PETrarCA, Canzoniere (a cura di M. Santagata), Milano: Mondadori, 1996, p. 1055, con il rimando al passo del Secretum dove, dopo aver menzionato la «litere velut pithagoree [...] doctrinam», Franciscus confessa: «Cum enim recto tramite ascendens ad bivium pervenissem modestus et sobrius, et dextram iuberer arripere, ad levam —incautus dicam an contumax? - deflexi [...]. Ex tunc autem obliquo sordidoque calle distractus et sepe retro lacrimans conversus, dextrum iter tenere non potui, quod cum deserui, tunc, profecto tunc, fuerat illa morum meorum facta confusio» (F. PETRARCA, Secretum, in Prose, cit., p. 150-2; naturalmente, come si legge nella nota esplicativa di p. 150, «la lettera pitagorica è l' $y$ che per la sua forma fu assunto nel Medioevo come simbolo della vita umana, nella quale a un certo punto s'aprono due vie, quella della virtù e quella del piacere». Ma si veda la spiegazione fornita dall'autore stesso nella familiare XII 3 «ad Zenobium grammaticum florentinum»: "Sentis, acutissime vir, [...] sentis illud grande discrimen, illud grave negotium quod intrantibus viam vite huius obicitur: longum iter asperumque, breve tempus et adversum; dexterior arduus angustus vepricosus scrupeus; ea nobis ad veram vitam semita est, at leva malorum / exercet pena set ad impia tartara mittit; quod nec Maro noster ignorat, nec Pythagoras ignorabat, dum Cadmi vestigiis insistens, scripture supervacuam sed vite utilem literam in incude ingenii mallearet. Bicornis et exemplaris litera dextro cornu arctior tendit ad siderea, levo latior in terram curvata reflectitur; ea, ut aiunt, ad inferos est via, et illa quidem incessu letior ac dulcior, exitu mestissima atque amarissima est, et cuius omnino nil possit miserie superaddi; dextrum vero iter ingressis ut labor ingens sic finis optimus» cit. per F. PETRARCA, Le Familiari, cit. p. 18-9).

5. E. FenzI, nota n. 98 al III libro, in F. Petrarca, Secretum (a cura di E. Fenzi), Milano: Mursia, 1992, p. 370.

6. J. Le Goff, La nascita del Purgatorio, Torino: Einaudi, 1996, p. 5. 
monte, investito del "sacro dell'“altitudine"», "punto d'incontro del cielo e della terra», 7 si è perfettamente insediato nel cuore della simbologia cristiano-medievale, fino alla montagna del Purgatorio dantesco con il suo percorso rituale di purificazione. Anche il Canzoniere è punteggiato di tensioni verso l'alto, di emblemi montani, di vie che conducono al cielo, di «ali» per spiccare il volo, che qui sarebbe superfluo esemplificare dettagliatamente. ${ }^{8} \mathrm{Ma}$ la «via de salir al ciel» $(R V F 68,4)$ — per citare solo una delle varianti espressive in cui si diffrange la grammaticalità del tema- diviene spesso la «dritta via di gir al ciel» ( $R V F$ 261, 7-8), il «camin destro di gire al ciel» $(R V F 306,1-2)$. Così, nel sonetto 25 al «dritto camin» del v. 5 risponde la terzina conclusiva col «...mostrar quanto è spinoso calle, / et quanto alpestra et dura la salita, / onde al vero valor conven ch'uom poggi». Insomma, simbolismo dell'alto e motivo del «bivio pitagorico» o della strada giusta che porta al bene ("l viaggio da la man destra, ch'a buon porto aggiunge» di $R V F 264,120-1)$ tendono a fondersi. ${ }^{9}$ Ed è questo stesso - doppio - regime metaforico a governare il racconto del Ventoso, ${ }^{10}$ con la correzione che all'alternativa magico-esoterica destra/sinistra apporta la più realistica opposizione, carica di implicazioni psicologiche, fra cammino comodo e impervio, deviazioni e scorciatoie, errare e puntare diritto alla meta:

Inde iterum digressi provehimur, sed lentius: et presertim ego montanum iter gressu iam modestiore carpebam, et frater compendiaria quidem via per ipsius iuga montis ad altiora tendebat; ego mollior ad ima vergebam, revocantique et iter rectius designanti respondebam sperare me alterius lateris faciliorem aditum, nec horrere longiorem viam per quam planius incederem. Hanc excusationem ignavie pretendebam, aliisque iam excelsa tenentibus, per valles errabam, cum nichilo mitior aliunde pateret accessus, sed et via cresceret et inutilis labor ingravesceret. Interea, cum iam tedio confectum perplexi pigeret erroris, penitus alta petere disposui ... (9-10).

7. «La montagna è più vicina al cielo, e questo le conferisce una doppia sacralità: da un lato partecipa al simbolismo spaziale della trascendenza ("alto", "verticale", "supremo", ecc.), e d'altra parte il monte è per eccellenza il dominio delle ierofanie atmosferiche. Ed è, in quanto tale, dimora degli dei. Tutte le mitologie hanno una montagna sacra, variante più o meno illustre dell'Olimpo. [...] Spesso la montagna è considerata punto d'incontro del cielo e della terra; quindi un "centro", punto per il quale passa l'Asse del Mondo, regione satura di sacro, luogo ove possono attuarsi i passaggi fra le zone cosmiche diverse. [...] L'altitudine ha una virtù consacrante. Le regioni superiori sono sature di forze sacre. Tutto quel che più si avvicina al cielo, partecipa con intensità variabile alla trascendenza. L“altitudine", il «superiore», sono assimilati al trascendente, al sovrumano. Ogni "ascensione” è una rottura di livello, un passaggio nell'oltretomba, un superamento dello spazio profano e della condizione umana. [...] $\mathrm{Ne}$ consegue che la consacrazione mediante rituali di ascensione o scalata di monti, o salita di scale, è valida perché inserisce chi la pratica in una regione superiore celeste» (M. ELIADE, Trattato di storia delle religioni, Torino: Boringhieri, 1976, p. 111-4).

8. Basti vedere le occorrenze segnalate da M. SANTAGATA nella nota al v. 13 di $R V F 13$, in F. Petrarca, Canzoniere, cit., p. 61.

9. E si fondono ad esempio, proprio sul piano della concentrazione enunciativa, nell' «ir dritto, alto" del sonetto 286, v. 9 (preceduto dal negativo della «man manca» al v. 8).

10. Secondo un'impostazione mutuata, come rileva N. MANN (op. cit., p. 12), da un brano del VI libro delle Divinae institutiones di Lattanzio. 
Nella «moralità» (quasi nel senso tecnico del genere) che va in scena sulle pendici del Ventoso, la divaricazione delle due strade, la strada facile del vizio e quella ardua della virtù, si traduce nella distribuzione delle parti tra i fratelli, che attivano in sincronia entrambe le possibilità del topos. Il cimento della salita fa risaltare a contrasto le loro diverse attitudini psicologiche e fisionomie morali. All' «iter rectius» di Gherardo (calco della «diritta via» smarrita da Dante) l'impianto comparativo della drammatizzazione contrappone gli svagati andirivieni del pigro Francesco, che, in cerca di un «faciliorem aditum», non esita ad allungare il tragitto pur di procedere "planius» e finisce per invertire la direzione tornando indietro, verso il basso («ad ima vergebam»). La filigrana allegorica della rappresentazione enfatizza, nel confronto con la risoluta aspirazione alla trascendenza del deuteragonista, l'«ignavia» con cui si muove invece, sotto il segno - dalla perspicua ambivalenza semantica - dell'"errore» ("per valles errabam», "perplexi erroris»), il protagonista, di continuo attirato a valle dalla fascinazione degli appetiti terreni. Il frastaglio dei suoi giri tortuosi sui fianchi della montagna è l'arabesco di una volontà impacciata, raffigura l'inconcludente attardarsi nella valle delle vanità mondane di chi indugia a incamminarsi per il sentiero ripido della virtù:

Cumque operientem fratrem [...] fessus et anxius attigissem, aliquandiu equis passibus incessimus. Vixdum collem illum reliqueramus, et ecce prioris anfractus oblitus, iterum ad inferiora deicior, atque iterum peragratis vallibus dum viarum facilem longitudinem sector, in longam difficultatem incido (10-1).

Ogni progresso è reversibile. Petrarca si ricongiunge al fratello e poi se ne separa di nuovo, si ravvede dell'errore ma poi vi ricade. Avanza e retrocede, sale e ridiscende. E intanto il tempo passa e la sommità del monte resta ancora lontana. Il tempo della vita si consuma nell'accidia, nel perpetuo rinvio della decisione gravosa di rinunciare ai piaceri del mondo («Differebam nempe ascendendi molestiam» [11]). Lo scrittore sta provvedendo anche qui a modellare l'idolo autobiografico della perplessità e dell'oscillazione, della scissione paralizzante dell'animo; che coinvolge, frontalmente, proprio la sfera della temporalità. Pure nella terza familiare del libro XII, ad esempio, da cui abbiamo citato in precedenza, al tema, per così dire, spaziale del «bivio pitagorico» si associa quello del tempo, del suo incalzare:

Sed vix unquam satis exacta custodia esse potest et pene quicquid cautionis aut diligentie adhibetur, minus est quam pro qualitate periculi: tot passim horrenda, tot perplexa, tot lubrica, tam multe dehortantium, tam multe detinentium ac retrahentium rerum forme. Atque utinam vel summa temporis parcitate et inutilibus sarcinis abiectis, inter tot adversa nudi expeditique quem destinavimus terminum, apprehendamus! ${ }^{11}$ 
Il cammino difficoltoso e accidentato verso il traguardo della salvezza deve fare i conti con la «summa temporis parcitas», è anche una gara contro l'imminenza della morte. Bisogna raggiungere la meta della purezza spirituale, il «terminus», finché si è in tempo. L'epistola del Ventoso - ne parlavamo all'inizio - abbonda di indicazioni e inquadrature cronologiche, culminanti in quella sorta di aritmetica della conversione che abbiamo già letto: «'Si tibi forte contingeret per alia duo lustra volatilem hanc vitam producere, tantumque pro rata temporis ad virtutem accedere quantum hoc biennio, per congressum nove contra veterem voluntatis, ab obstinatione pristina recessisti, nonne tunc posses, etsi non certus at saltem sperans, quadragesimo etatis anno mortem oppetere et illud residuum vite in senium abeuntis equa mente negligere?’”. Non importa tanto rilevare come questo Petrarca che matematizza le tappe della sua redenzione secondo un diagramma di incrementi costanti e scadenze precise (quasi alla maniera di un mercante che stili un bilancio di previsione), partecipi anch'egli dell'onda ormai abbastanza lunga di quella «mentalità aritmetica" la cui genesi nel tardo Medioevo è stata ricostruita da Murray. ${ }^{12}$ Importa di più la nozione del tempo che questo reticolo di calcoli implica. Se "con il Purgatorio", come scrive Le Goff alludendo al titolo seducente di un saggio di Chiffoleau, addirittura «si apre la contabilità dell'aldilà», mentre "prima c'erano soltanto l'eternità o l'attesa indeterminata», ${ }^{13}$ questo tempo dell'anima così esattamente calcolato, misurato, scandito nella lettera petrarchesca è il tempo di un progetto umano, un tempo da preventivare e usare come una proprietà dell'io, come una grandezza le cui proporzioni tutte terrene sono configurate dalle stagioni della "voluntas». Poiché il tempo è un bene preziosissimo da amministrare con la massima avvedutezza, ${ }^{14}$ anche la cristiana cura della coscienza può ormai ritmarsi secondo il «tempo del mercante», inscrivendosi nella nuova orbita mentale laicizzante per sviluppare quella che si potrebbe definire «una morale calcolatrice». ${ }^{15}$ Naturalmente, in una tale cali-

12. A. Murray (Ragione e società nel Medioevo, Roma: Editori Riuniti, 2002) afferma che è nell'Italia del Duecento che «l'invasione dei numeri» comincia a manifestarsi anche nel campo della letteratura, specchio privilegiato di ogni trasformazione culturale, dove si può dunque cogliere bene «la misura dell'influenza della matematica sul pensiero non-specialistico». E documento massimo di queste mutate «abitudini mentali dei letterati» sarà il "carico quasi ingombrante di matematica» presente nella Commedia dantesca (le citazioni si riferiscono alle p. 185 e 194; ma si veda tutta la parte seconda del libro intitolata L'aritmetica).

13. J. Le Goff, La nascita, cit., p. 258. Infatti, «ora si computa il tempo di Purgatorio a seconda della gravità dei peccati e il tempo di condono di Purgatorio a seconda dell'importanza dei suffragi, si calcola il rapporto tra il tempo vissuto qui e quello di cui si prova la sensazione laggiù» (ibidem).

14. Si pensi, ad esempio, alla familiare XVI 11 «Ad Franciscum Sanctorum Apostolorum, quam cara res sit tempus».

15. "La scossa del quadro cronologico conosciuta dal secolo XIV è anche una scossa mentale, spirituale. Forse bisognerebbe cercare nella scienza medesima, cioè nella scolastica scientifica, l'apparizione di una nuova concezione del tempo, di un tempo che non è più un'essenza, ma una forma concettuale, al servizio dello spirito che ne usa secondo i propri bisogni, può dividerlo, misurarlo: un tempo discontinuo. [...] Un tempo nuovo si profila nella scola- 
bratura di articolazioni temporali, come, per esempio, nell'insistenza con cui, qui e altrove, la mutatio animi è situata intorno ai quaranta anni, permane una robusta componente allusiva e simbolica; ${ }^{16}$ che non mette in questione, però, il senso di questa appropriazione umana del tempo: non il tempo assoluto di Dio ma il tempo come misura interna dell'esistenza, la chiave di cui l'uomo si serve per interpretarla, darle valore, orizzontarvisi. Né, d'altra parte, arrivano a incrinare un simile modo di sentire il tempo la sottolineatura della fragilità della vita («Si tibi forte contingeret per alia duo lustra volatilem hanc vitam producere...») e il gravitare del soliloquio verso lo sbocco supremo della morte, giacché "gli uomini del Rinascimento», che Petrarca annuncia, «sono padroni del loro tempo come del resto. Solo la morte li limita, ma colta [...] in una prospettiva del tutto nuova, in cui la fine diventa il punto di partenza della riflessione e in cui la decomposizione corporale suscita il senso della durata, come ha mostrato Alberto Tenenti attraverso analisi nuove delle «artes moriendi» e del pensiero umanistico francese e italiano». ${ }^{17}$ Nello stesso accento posto come non mai sulla centralità della morte ${ }^{18}$ («Intorno al 1350 il sentimento della morte stava divenendo un cardine della vita spirituale [...] attraverso l'intensificarsi del senso della distruzione fisica", così familiare all'autore del Secretum) vibra la "volontà ostinata della vita di imporsi come valore autonomo» testimoniata dagli umanisti, la cui «arte di ben morire era in fondo il nuovo senso del tempo, del valore del corpo come organismo, e si risolveva in un ideale di vita attiva che non aveva più il centro di gravità al di fuori dell'esistenza terrestre». ${ }^{19}$

stica nello stesso momento in cui gli studi sull'impetus rivoluzionano la meccanica, e in cui la prospettiva moderna comincia a sconvolgere la visione. Il secolo dell'orologio è anche quello del cannone e della profondità di campo. Tempo e spazio per lo scienziato, come per il mercante, si trasformano insieme. Forse, anche il tempo dei mistici, dei grandi mistici renani anzitutto, è il frutto di un nuovo approccio, di una nuova intuizione, che dà alla vita dell'anima nuove dimensioni temporali. [...] In ogni caso a livello di una devozione più accessibile, più media, la scossa è netta. Il tema eterno, antico, della fuga del tempo si era ritrovato nel cristianesimo, esasperato e insieme placato dalla sua trasformazione in timore della morte eterna, incitamento alla preparazione della salvezza. "Nihil pretiosius tempore", avrebbe detto san Bernardo, e questo tema è in ogni caso ripreso e diffuso dai suoi discepoli. Ma, fin dalla prima metà del secolo XIV, il tema si precisa, si drammatizza. Perdere il proprio tempo diviene un peccato grave, uno scandalo spirituale. Sul modello del denaro, per imitazione del mercante, che, almeno in Italia, diventa un contabile del tempo, si sviluppa una morale calcolatrice, una forma avara di pietà» (J. Le GofF, Tempo della Chiesa e tempo del mercante, Torino: Einaudi, 1977, p. 36-7).

16. V., ad esempio, M. SANTAGATA, I frammenti dell'anima (Storia e racconto nel Canzoniere di Petrarca), Bologna: Il Mulino, 1992, p. 80-3. Ma in Petrarca il simbolismo religioso dei numeri e delle date sembra comunque avviato verso una specie di secolarizzazione, come mostra l'ordinamento profano dell'autobiografia del Canzoniere, in cui le scansioni della storia d'amore collimano, in ambigua antitesi, con le grandi ricorrenze del calendario liturgico.

17. J. Le Goff, Tempo della Chiesa, cit., p. 22.

18. E «il Purgatorio è una delle cause essenziali della drammatizzazione del momento della morte» (J. Le GofF, La nascita, cit., p. 331).

19. A. TENENTI, Il senso della morte e l'amore della vita nel Rinascimento (Francia e Italia), Torino: Einaudi, 1977, rispettivamente p. 40, 34, 63. 
Ma, a onta dell'istanza finalistica da cui sembra mossa, l'epistola del Ventoso dice soprattutto che Petrarca sconfessa il tempo teologico del fine, e della fine, il tempo orientato in linea retta verso il telos della salvezza, ${ }^{20}$ per sostituirvi la tortuosità esistenziale dei desideri, delle incertezze, delle resistenze, delle tentazioni risorgenti, delle ricadute. E' questo il vero tempo umano; un tempo vischioso, ripetitivo, curvilineo, sinuoso, reversibile. E quindi un tempo lento, al quale resta essenzialmente estranea la sovrannaturale fulmineità del tempo della grazia. L'apertura casuale delle Confessioni («Aperio, lecturus quicquid occurreret» [26]) trafigge sulla vetta il protagonista con una folgorazione conoscitiva che appartiene al tempo sacro della rivelazione. Nel momento epifanico della bibliomanzia il caso coincide magicamente con la necessità, la contingenza con l'assoluto. Il lampeggiamento istantaneo della verità è avvalorato dalla violenza con cui turba nel profondo lo scrittore, lo annichilisce nell'atto stesso di illuminarlo: «Obstupui, fateor» (28). Ma dopo lo «stupore», ${ }^{21}$ ovvero lo smarrimento, quasi l'estasi della commozione mentale, il tempo umano dell'andirivieni e dell' «errore», corrispondente al vagare nello spazio in questo eloquentissimo cronotopo dell'ascensione al monte, riprende il suo corso intricato, tortuoso, letteralmente "perplesso». Realtà fisica e realtà interiore si compenetrano esemplarmente, a riprova della straordinaria compattezza figurale della scrittura, nell'epilogo, che non può che essere una preghiera innalzata all'eterna perfezione divina da colui che continua il cammino errante e instabile dell'umano:

Vide itaque, pater amantissime, quam nichil in me oculis tuis occultum velim, qui tibi nedum universam vitam meam sed cogitatus singulos tam diligenter aperio; pro quibus ora, queso, ut tandiu vagi et instabiles aliquando subsistant, et inutiliter per multa iactati, ad unum, bonum, verum, certum, stabile se convertant (36).

L'intuizione della retta via non ha la perentorietà di una conversione; segna appena l'inizio di un lungo itinerario penitenziale. Si potrebbe sostenere che per Petrarca esistano due tempi differenti, un tempo — eventualmente anche immediato, epifanico, come si diceva - dell'intelligenza, della visione intellettuale delle cose, e il tempo lento, resistente, appiccicoso, faticoso della persuasione intima, della conoscenza che si fa carne e nervi, che diviene ispirazione profonda della coscienza, regola immanente dei pensieri e delle decisioni. Vedere il bene non significa essere subito in grado di praticarlo. Il «viaggio da la man destra» è rallentato dalle mille insidie della quotidianità, dall'inerzia dei giorni e dei loro accadimenti. "Veggio 'l meglio, et al peggior m'appiglio» ( $R V F$ $264,136)$. D'altro canto, secondo Petrarca il sapere autentico è quello incar-

20. Per la particolare prospettiva escatologica che distingue il cristianesimo dall'ebraismo v., in estrema sintesi, J. Le Goff, Tempo della Chiesa, cit., p. 6-7.

21. Sul motivo ambivalente dello «stupore», che in Petrarca fondamentalmente rappresenta lo stigma dell'alienazione amorosa, v. E. FENZI, nota n. 105 al III libro, in F. PETRARCA, Secretum, cit., p. 370-1. 
nato, radicato nell'esperienza individuale. «Esperienza» è parola chiave e Leitmotiv concettuale tra i più spiccanti del Secretum, dove l'invenzione preliminare del silenzio della Verità riconduce l'intensità della meditazione al piano dell'esperienza, appunto, a un dialogo fra interlocutori umanamente ancorati al fondo martoriato del loro vissuto. "Expertus loquor», dice Agostino. ${ }^{22}$ Per trasformarsi in nutrimento dell'animo ogni acquisizione culturale, ogni scoperta dell'intelletto ha bisogno di maturare lentamente alla prova della vita. Nel brano del Secretum che abbiamo già addotto a proposito della «lettera pitagorica» Francesco ricorda di avere ripetutamente letto i versi virgiliani sulla via che si biforca nell'oltretomba ("Hic locus est partes ubi se via findit in ambas...»), senza però giovarsene o capirli davvero («neque michi profuit quod sepe puer legeram») prima di sperimentarne di persona il senso negli ondeggiamenti della propria volontà: "Hec nimirum, quanquam ante legissem, non tamen prius intellexi quam expertus sum». ${ }^{23}$ Anche l'ammonimento che sul Ventoso erompe subitaneo dalle parole di Agostino dovrà assoggettarsi al tempo ingorgato della volontà perplessa.

\section{II}

L'ascesa del monte come simbolo di ascesi (secondo il veridico suggerimento della paronomasia) è il perno sul quale ruota la figuralità dell'epistola. Ed è cifra non solo dispiegata con una diffusione che la rende lampante ma, quasi non fosse abbastanza chiara al lettore, addirittura messa dal testo autoriflessivamente in rilievo, ${ }^{24}$ esplicitata attraverso una pausa meditativa dell'io narrante nella sua funzione di personaggio:

Sic sepe delusus quadam in valle consedi. Illic a corporeis ad incorporea volucri cogitatione transiliens, his aut talibus me ipsum compellabam verbis: «Quod totiens hodie in ascensu montis huius expertus es, id scito et tibi accidere et multis, accedentibus ad beatam vitam; sed idcirco tam facile ab hominibus non perpendi, quod corporis motus in aperto sunt, animorum vero invisibiles et occulti. Equidem vita, quam beatam dicimus, celso loco sita est; arcta, ut aiunt, ad illam ducit via. Multi quoque colles intereminent et de virtute in virtutem preclaris gradibus ambulandum est; in summo finis est omnium et vie terminus ad quem peregrinatio nostra disponitur. Eo pervenire volunt omnes, sed, ut ait Naso, Velle parum est; cupias, ut re potiaris, oportet. Tu certe — nisi, ut in multis, in hoc quoque te fallis - non solum vis sed etiam cupis. Quid ergo te retinet? nimirum nichil aliud, nisi per terrenas et infimas voluptates planior et ut prima fronte videtur, expeditior via; veruntamen, ubi multum erraveris,

22. F. Petrarca, Secretum, in Prose, cit., p. 100.

23. Ivi, p. 150.

24. «La lettera» — scrive B. MartinelLi («Petrarca e l'epistola del Ventoso: i diversi tempi della scrittura», Rivista di letteratura italiana, n. 19, 2001, p. 57) — «è insieme una narrazione e una confessione; e in quanto non ci dà solo il resoconto dei fatti e non si limita a presentarli al suo destinatario, ma li interpreta e ne fissa le chiavi della lettura, è anche un testo di tipo diverso, è un récit ermeneutico». 
aut sub pondere male dilati laboris ad ipsius te beate vite culmen oportet ascendere aut in convallibus peccatorum tuorum segnem procumbere; et si — quod ominari horreo - ibi te tenebre et umbra mortis invenerint, eternam noctem in perpetuis cruciatibus agere» (12-5).

Il personaggio accidioso che stenta a dirigersi di buona lena verso la cima illustra puntualmente a sé stesso il significato nascosto della situazione in cui si trova, ponendo in relazione ciò che gli sta materialmente accadendo con il suo etimo spirituale, $\mathrm{i}$ "corporea» con gli «incorporea». Può apparire una ridondanza intellettualistica, che rischia magari di pregiudicare, ai nostri occhi di moderni, l'equilibrio diegetico tra accidentalità dei fatti e astrazione allegori$\mathrm{ca}$; ma, a guardare più addentro, questo squarcio metasimbolico ${ }^{25}$ è forse, paradossalmente, la spia di una crisi incipiente della rappresentazione simbolica del mondo propria della civiltà del Medioevo. Vale a dire che la «simbologia, intesa [...] come linguaggio specifico e insostituibile della mentalità medievale» ${ }^{26}$ (per usare le parole di un grande studioso che fu, in anni ormai lontani, tra i più energici rivendicatori della medievalità quale peculiare, irriducibile sistema di misure culturali) sembra qui inclinare piuttosto, nello stesso risalto autoriflessivo, alla calibrata letterarietà di una similitudine che, per giunta, mentre li connette, insiste tuttavia ad evidenziare la distinzione tra $\mathrm{i}$ "corporis motus», che «in aperto sunt», e i moti dell'animo «invisibiles et occulti», le entità per essenza inconciliabili del corpo e dello spirito, collegate dalla cogitatio del soggetto ma in definitiva irrimediabilmente divaricate, ${ }^{27}$ come ter-

25. Altra cosa è il rigore ontologico di certe spiegazioni dantesche, come quando, per chiarire la presenza costante del numero nove nella vita di Beatrice, il poeta afferma che «questo numero fue ella medesima» e che "questa donna fue accompagnata da questo numero del nove a dare ad intendere ch'ella era uno nove, cioè uno miracolo, la cui radice, cioè del miracolo, è solamente la mirabile Trinitade» (Vita nuova, XXIX, 3-4).

26. S. BATTAGLia, op. cit., p. 52. Si sa, del resto, che «l'importanza, pur indubitabile, che l'allegoria retorica ha nel Medioevo, svanisce quasi in confronto con quella dell'allegoria esegetica. L'allegoresi può veramente essere definita la componente-chiave della forma mentis medievale, essendo la base stessa del modo in cui il Medioevo concepisce sia la realtà terrena sia quella escatologica e si rapporta ad esse. In linea con un approccio esegetico antico quanto il Cristianesimo stesso, l'allegoresi viene percepita come lo strumento attraverso cui è possibile qui sulla terra cogliere un senso della volontà divina. E' un luogo comune del pensiero medievale che la realtà, in quanto prodotto della creazione divina, è tutt'altro che arbitraria. Perciò, sotto la superficie delle cose (res) e degli eventi (bistoria), Dio ha provvidenzialmente inserito una serie di significati (signa) che non solo offrono, per analogiam, la possibilità di penetrare, sia pur pallidamente e in via provvisoria, nella natura di Lui e della vita oltremondana (sensus anagogicus), ma indicano anche chiaramente che Dio stesso ha organizzato l'intero essere in modo tale da favorire la salvezza spirituale dell'umanità (sensus allegoricus)» (Z.G. BARAÑSKI, «La "Commedia”", in F. BRIOSCHI-C. Di GIROLAMO (a cura di), Manuale di letteratura italiana. Storia per generi e problemi, I. Dalle origini alla fine del Quattrocento, Torino: Bollati Boringhieri, 1993, p. 496).

27. "Questo passo è dominato dalla differenza fra il corporeo e l'incorporeo: la parola transiliens esprime già la presenza di una spaccatura fra i due, che significa anche una spaccatura fra il fatto empirico e il significato allegorico» (R.M. DURLING, «Il Petrarca, il Ventoso e la possibilità dell'allegoria», Revue des études augustiniennes, n. 23, 1977, p. 308). 
mini di un parallelismo artificialmente costruito che finisce per cedere il posto alla differenza:

Hec michi cogitatio incredibile dictu est quantum ad ea que restabant et animum et corpus erexerit. Atque utinam vel sic animo peragam iter illud, cui diebus et noctibus suspiro, sicut, superatis tandem difficultatibus, hodiernum iter corporeis pedibus peregi! Ac nescio annon longe facilius esse debeat quod per ipsum animum agilem et immortalem sine ullo locali moto in ictu trepidantis oculi fieri potest, quam quod successu temporis per moribundi et caduci corporis obsequium ac sub gravi membrorum fasce gerendum est (15).

La meccanica terrestre del corpo non è comparabile davvero, malgrado il gioco delle equivalenze, alla libertà dell'anima immortale, che può congiungersi a Dio in un batter d'occhio. "Tutto il cosmo era per il Medioevo un cifrario dello spirito»; ${ }^{28}$ ma ora il viandante che sale per i sentieri della montagna e lo spirito che tende alla vita beata possono sovrapporsi soltanto in un'analogia imperfetta.

Ma un'effettiva, inequivocabile, perfino fragorosa rottura del codice simbolico è quella che si consuma, in maniera irreversibile, allorché Petrarca si trova finalmente insieme al fratello sulla vetta più alta; alla quale applica innanzitutto, e sintomaticamente, la competenza dell'etimologista ("Collis est omnium supremus, quem silvestres "Filiolum" vocant; cur, ignoro; nisi quod per antifrasim, ut quedam alia, dici suspicor: videtur enin vere pater omnium vicinorum montium» [16]), quasi a isolare il mondo nella sua realtà verbale, il mondo come storia di parole e stratificazione di significati e discorsi umani, di cui spetta alla filologia, anziché all'allegorismo, l'interpretazione autentica. Poi, dopo aver ribadito il tema ascensionale con lo squillo di una «figura etimologica» che declina, quindi, la coincidenza di fisico e morale in forma di artificio retorico, e mentre - condensandosi il tempo dei fatti in quello della lettural'evento sembra già, coerentemente, ridisporsi nella latitudine della finzione letteraria («Et quoniam audiisti quenam ascendentis in pectus ascenderint cure, audi, pater, et reliqua; et unam, precor, horam tuam relegendis unius diei mei actibus tribue» [ibidem]), l'autore passa a registrare le reazioni provocategli dalla maestà dello spettacolo che gli si era presentato. Alla vertigine iniziale dello «stupore» (e abbiamo già accennato alla rilevanza di questo elemento), come dinanzi a una ierofania, subentra subito, di nuovo, l'orizzonte memoriale della letteratura e della storia:

Primum omnium spiritu quodam aeris insolito et spectaculo liberiore permotus, stupenti similis steti. Respicio: nubes erant sub pedibus; iamque michi minus incredibiles facti sunt Athos et Olimpus, dum quod de illis audieram et legeram, in minoris fame monte conspicio. Dirigo dehinc oculorum radios ad partes italicas, quo magis inclinat animus; Alpes ipse rigentes ac nivose, per quas ferus ille quondam hostis romani nominis transivit, aceto, si fame credimus, 
saxa perrumpens, iuxta michi vise sunt, cum tamen magno distent intervallo. Suspiravi, fateor, ad italicum aerem animo potius quam oculis apparentem, atque inextimabilis me ardor invasit et amicum et patriam revidendi, ita tamen ut interim in utroque nondum virilis affectus mollitiem increparem, quamvis excusatio utrobique non deforet magnorum testium fulta presidio (17-9).

I primi pensieri che la sosta «in vertice» sollecita sono, dunque, pensieri terreni. Invece che la patria celeste ${ }^{29}$ a cui l'ha fisicamente e simbolicamente avvicinato, l'altezza del luogo richiama a Petrarca la patria italiana. E' una nostalgia colpevolmente umana («ita tamen ut interim in utroque nondum virilis affectus mollitiem increparem») la più immediata ripercussione mentale («ad italicum aerem animo potius quam oculis apparentem») dell'ascesa. Poi, però, il filo delle riflessioni ha uno strappo, che lo scrittore commenta con la consueta lucidità, con una sorta, ancora, di sigla metanarrativa: «Occupavit inde animum nova cogitatio atque a locis traduxit ad tempora» (19); e comincia il soliloquio doloroso dell" "uterque homo» che già conosciamo («Dicebam enim ad me ipsum...»), con il suo intreccio, tipicamente petrarchesco, di ricordo e speranza, di passato e futuro: «Sic per exactum decennium cogitatione volvebar. Hinc iam curas meas in anteriora mittebam...» (22-3). Ma più avanti la serie delle «cogitationes» morali, che sembravano perfettamente intonate alla condizione di un'anima innalzatasi al di sopra della bassura delle cose mondane, è interrotta proprio perché non sarebbero questi il momento e il luogo adatti:

De provectu meo gaudebam, imperfectum meum flebam et mutabilitatem comunem humanorum actuum miserabar; et quem in locum, quam ob causam venissem, quodammodo videbar oblitus, donec, ut omissis curis, quibus alter locus esset oportunior, respicerem et viderem que visurus adveneram [...], verto me in tergum, ad occidentem respiciens (24-5).

In altri termini: lo spessore simbolico viene bruscamente cancellato, oscurato a beneficio del livello letterale, dell'autonomia dell'episodio, ovvero della cupiditas naturalistica dichiarata fin dall'esordio nella sua esclusività: "Altissimum regionis huius montem, quem non immerito Ventosum vocant, hodierno die, sola videndi insignem loci altitudinem cupiditate ductus, ascendi» (1). Allora lo sguardo, un inaudito sguardo dall'alto, ${ }^{30}$ spazia sul panorama sotto-

29. Quella di cui parla, tanto per fare un esempio, il finale di Fam. XIV 1: «Difficultates cuntas facile superabis, si exilii molestiam brevitate consolans, semper suspires ad patriam cui, si placet, ascriptus es civis, ubi sicut optima sic eterna sunt omnia» (F. PetrarCA, Le Familiari, vol. III, cit., p. 105).

30. Si ricordi che "per l'Antichità, proprio come per il Medioevo, vi è una singolare inibizione a contemplare il mondo dall'alto o a immaginarlo contemplato dall'uomo in questo modo. Il soggiorno naturale dell'uomo è in basso, e la direzione costitutiva del suo sguardo è dal basso verso l'alto, è quella del contemplator caeli. [...] Lo sguardo dall'alto sul mondo è riservato agli dei $[\ldots]$. In pittura la conquista della prospettiva dall'alto è una delle innovazioni dell'inizio dell'età moderna» (H. BluMENBERG, La legittimità dell'età moderna, Genova: Marietti, 1992, p. 367 n. 223). 
stante, ${ }^{31}$ dai monti della provincia lionese al mare di Marsiglia e al Rodano. Ed è uno sguardo che recupera, tuttavia, la sublimità dell'occasione: "Que dum mirarer singula et nunc terrenum aliquid saperem, nunc exemplo corporis animum ad altiora subveherem...» (26). Nella dicotomia di «terrenum»e «altiora» le caratteristiche fluttuazioni della coscienza, chiamata ad adeguarsi all' "esempio del corpo", mettono a nudo per l'ennesima volta, nitidamente, il regime connotativo del testo. A questo punto l'ultima virata. La vicenda precipita verso lo scioglimento folgorante che sappiamo: «Visum est michi Confessionum Augustini librum, caritatis tue munus, inspicere» (ibidem). E la "cupiditas videndi» appena riproposta incorre nella condanna agostiniana: «Et eunt homines admirari alta montium et ingentes fluctus maris et latissimos lapsus fluminum et occeani ambitum et giros siderum, et relinquunt se ipsos». Ma le parole di Agostino non comportano soltanto una svalutazione del sapere scientifico della scolastica in favore dello sprofondamento dentro la soggettività della filosofia morale; esse vengono anche a scompigliare l'ordine delle corrispondenze simboliche e delle connotazioni assiologiche che sorreggevano l'epistola. Se gli «alta montium» fanno parte dello scenario esteriore del mondo che spossessa l'uomo della consapevolezza di sé, l'altitudine non ha più, per dirla con Eliade, una virtù consacrante. Anche il desiderio di andare ad ammirare gli alti monti è una passione mondana, ${ }^{32}$ appunto come quelle dalle quali la salita sul Ventoso doveva allegorizzare la liberazione. Il simbolismo dell'alto, dell'ascesa è sottratto adesso al suo significato di purificazione interiore e di innalzamento dell'animo a Dio, così esplicitamente ribadito in precedenza, per essere collegato, con un inopinato slittamento, alla negatività ${ }^{33}$

31. Trasgredendo la percezione medievale di un mondo gerarchizzato: «Proliferano nel discorso sociale [del Medio Evo] i modelli luminosi, gli schemi ascensionali, le immagini di apertura e di slancio spaziale: la mistica cristiana a partire da Bernardo di Chiaravalle si esprime in termini di elevazione; Ugo di San Vittore [...] assimila alto e virtù, basso e vizio. La regalità si identifica con la verticalità dello scettro; il tipo della scala si applica alla Croce salvatrice, scala dei peccatori, e rende conto dei progressi dell'ascesi e del cammino delle virtù [...]. Ma non si tratta tanto di strutture stabilizzate quanto di un incessante movimento processionale, dall'alto in basso e, all'inverso, di un nomadismo della verticalità, secondo il quale la disuguaglianza delle cose e degli esseri appariva come una forma dell'armonia e le differenze di grado quali dati naturali non aggirabili. [...] Si costituisce così un universo di immagini e di luoghi comuni, che sopravvivrà in parte sino a noi, mentre le grandi scoperte del XV e XVI secolo, eliminando il rischio del meraviglioso, avranno paradossalmente finito per bloccare il nomadismo dello spirito. Forse questo risultato era stato annunziato, fin dalla metà del XIV secolo, da un testo come l'epistola in cui Petrarca, narrando la sua ascensione del monte Ventoso, contemplava, da questa altezza che faceva sua, il mondo appiattito" (P. ZUMTHOR, La misura del mondo (La rappresentazione dello spazio nel Medio Evo), Bologna: Il Mulino, 1995, p. 34-5).

32. La "cupiditas videndi», pertanto, deve trasferirsi al mondo interiore: «Tunc vero montem satis vidisse contentus, in me ipsum interiores oculos reflexi» (29).

33. Né manca il topico attributo negativo dell'altezza come superbia: «Si tantum sudoris ac laboris, ut corpus celo paululum proximius fieret, subire non piguit, que crux, quis carcer, quis equuleus deberet terrere animum appropinquantem Deo, turgidumque cacumen insolentie et mortalia fata calcantem?» (33-4) 
della curiositas scientifica. E' una sorpresa, una rotazione incongruente del congegno allegorico che, tra l'altro, a voler essere troppo consequenziari, arriva perfino a proiettare un'ombra, retrospettivamente, sulla dirittura di Gherardo, sul fervore della sua aspirazione alla «vita beata» — che «celso loco sita est»—, sminuendone in qualche modo l'esemplarità. E d'altronde, a tale riguardo, proprio questa lettera ${ }^{34}$ comprova che la responsabilità ideale di cui Petrarca intende caricare la propria esperienza autobiografica non è quella di emblematizzare, come si dice per Dante, la storia di Ognuno, di Everyman nel suo pellegrinaggio sulla terra, bensì di accreditare l'esemplarità, con i suoi caratteri complessi e affatto particolari, dell'intellettuale umanista che affronta per primo la problematica ideologica di un'epoca di transizione.

$\mathrm{Ma}$, soprattutto, questa perturbazione dell'intelaiatura figurale dimostra che «le tensioni più vitali della cultura e dello stile petrarcheschi conducevano irresistibilmente verso una trasformazione dall'interno e un superamento delle forme allegoriche tradizionali», riconvocate sulla scena del testo, con freddezza metaletteraria, come per una cerimonia d'addio:

L'ascesa verso la vetta si presenta inizialmente come un'allegoria del difficile itinerario dell'uomo verso la beatitudine. Come nella lettera a Gherardo sulla poesia allegorica, la chiave interpretativa è resa esplicita dallo stesso Petrarca [...]. Ma, nel suo stesso svilupparsi, questo schema allegorico incontra la necessità del proprio superamento, per aprirsi all'epifania di quelle occulte realtà interiori cui esso rinvia e che costituiscono il vero centro della riflessione petrarchesca. Giunto infatti sulla cima del monte - che nella iniziale prospettiva allegorica rappresentava il raggiungimento della beatitudine- Francesco legge un brano delle Confessioni in cui sant'Agostino invita a distogliere lo sguardo dagli spettacoli esteriori per volgerlo dentro di noi: allora lo stesso panorama alpino che fino a quel momento aveva costituito la proiezione simbolica del cammino spirituale dell'anima diventa a sua volta un ostacolo, uno schermo che impedisce il ripiegamento meditativo su si sé, la vera conquista dell'interiorità. [...] A contatto con la nuova visione umanistica del Petrarca, la scrittura allegorica si rivela perciò infine inadeguata, lasciando il posto a una autoanalisi psicologica che troverà la sua espressione più compiuta nelle liriche italiane. ${ }^{35}$

34. In essa, come dice Durling, «siamo di fronte alla questione della possibilità di vivere, e d'interpretare la propria esperienza, nei termini universali fornitici dalla tradizione» (op. cit., p. 309).

35. F. ZAMBON, «La letteratura allegorica e didattica. 3. Il Tre e Quattrocento», in F. BRIOSCHIC. Di Girolamo (a cura di), Manuale di letteratura italiana, I, cit., p. 566-7. Su un versante contiguo, la consueta finezza critica di Adelia NOFERI intravede nella senile IV 5, che insinua una "totale relativizzazione dei significati nel testo", «'inizio di una svolta epistemologica: l'allegoria entra in una crisi profonda, proprio perché viene a mancare il congegno della sua "macchina", la funzione specifica e fondamentale di trattenere, codificare, ridurre e dominare l'ambiguità polisemica del linguaggio e la deriva del senso", dal momento che «la cultura medievale, con la vasta e protratta elaborazione teorica dell'allegoria, [...] aveva organizzato il processo di significazione limitando in estensione il numero dei significati, distribuendoli a strati ed a livelli gerarchicamente sovrapposti, ancorandoli saldamente ad una assoluta oggettività e ad una assoluta verità: quella di Dio, della parola e della scrittura di Dio» (A. NOFERI, «La senile IV 5: crisi dell'allegoria e produzione del senso», Quaderni petrarcheschi, n. 9-10, 1992-93, rispettivamente p. 695, 691 e 689). 
La smentita inflitta alla significazione spirituale della fenomenologia del mondo, il divorzio tra il "corporeo» e l' «incorporeo» ${ }^{36}$ sanziona l'eclissi del simbolismo nel suo valore conoscitivo di fulcro della rappresentazione teologico-medievale della realtà. Il cosmo cessa di apparire un cifrario dello spirito: nell'antinaturalismo petrarchesco la natura, non più simulacro del divino, si profila, più o meno implicitamente, come un oggetto autonomo di interesse e di scienza. Il monte riguadagna la sua individualità geografica, mentre sul piano simbolico viene umiliato -e l'abbiamo visto- dall'antropocentrismo della nuova Weltanschauung: "vix unius cubiti altitudo visa est pre altitudine contemplationis humane». L'antitesi semiotica interno vs esterno, che sostituisce nella «misura del mondo" quella alto vs basso, attribuisce al pensiero umano il compito di rimodellare l'immagine delle cose. E questo sfaldarsi dell'organicità teologica del reale si riflette sul simbolismo operante nella produzione del lirico volgare, che sarà spesso un simbolismo profanamente privato, soggettivistico, ${ }^{37}$ come, ad esempio, quello numerologico del Canzoniere (sul quale non occorre soffermarsi) incentrato sul numero 6, quasi un senhal aritmetico di Laura, e quindi sprovvisto della validità ontologica, ad esempio, del ritmo ternario soggiacente alla Commedia $;^{38}$ mentre per le poesie di più accentuato stampo allegorico dei Rerum vulgarium fragmenta si può parlare piuttosto di "tendenza all'emblematismo», ${ }^{39}$ di un cristallizzarsi di figurazioni predilette la cui costanza allusiva si adempie e giustifica all'interno di una mitografia tutta personale.

36. E si veda, al par. 34, l'ulteriore sfasatura tra coscienza e oggettività naturale, che svuota ancora l"alto' della sua pregnanza simbolica: «O quanto studio laborandum esset, non ut altiorem terram, sed ut elatos terrenis impulsibus appetitus sub pedibus haberemus!».

37. Così, parallelamente, il problema dell'interpretazione si sposterà «dalla oggettività metafisica medievale e dantesca alla soggettività del lettore» (A. NOFERI, op. cit., p. 695).

38. Dove sussiste, infatti, una «stretta relazione» tra "Dio creatore dell'Universo e Dante creatore della Comedía costruita, come il mondo, secondo criteri geometrici, numerologici e perciò armonici a riflettere, seppur imperfettamente, l'armonia del creato» (R. MERCURI, "Comedía” di Dante Alighieri», in A. Asor RosA (a cura di), Letteratura italiana. Le Opere. I. Dalle Origini al Cinquecento, Torino: Einaudi, 1992, p. 216).

39. F. ZAMBON, op. cit., p. 568: "La visione di Laura - referente ultimo, limite o margine del discorso- sembra irrompere sulla scena allegorica fino a dissolverla o a condensarla in cornice emblematica. E' il caso, particolarmente, della grande canzone CXXIX, il cui incipit $(D i$ pensier in pensier, di monte in monte) — quasi un titolo - indica programmaticamente i due livelli di significazione del testo: lo schema è analogo a quello messo in opera nel racconto dell'ascensione al Ventoso; qui però la simbologia alpina è riferita non più all'itinerario spirituale dell'anima, ma a una sorta di "ascesi” amorosa. E come nella lettera a Dionigi, il paesaggio montano a poco a poco si "desimbolizza”, trasformandosi da allegoria dell'esperienza interiore in ostacolo che separa e nasconde: Laura è lontana, oltre i monti/pensieri per i quali vaga il poeta. Ma, nello stesso tempo, lo schermo naturale è anche lo sfondo che dischiude il fantasma dell'amata: "et pur nel primo sasso / disegno co la mente il suo bel viso" (v. 28-29). Monti, colli e selve sono diventati frammenti di un paesaggio emblematico che tende a formare una sorta di sistema chiuso, soggetto alle regole di un'arte combinatoria interna». 


\section{III}

La familiare XVI 14, riprendendo quello che si potrebbe definire il «motivo taleteo» (si ricordi «la caduta del protofilosofo» studiata da Blumenberg) del Ventoso - gli uomini di cultura, tutti immersi ambiziosamente nelle occupazioni delle loro discipline, trascurano ciò che solo importa, la propria vita mora$1 \mathrm{e}^{40}$ _ , enuncia la suggestiva metafora dei «numeri ac mensure anime» («Sileo arythmeticos ac geometras, qui omnia numerant, omnia metiuntur, unius anime numeros ac mensuras negligunt»). ${ }^{41}$ Sigillo dell'agostinismo petrarchesco, le «misure dell'anima» sono la formula della conoscenza vera e utile, che bisogna curarsi di approfondire più di ogni altro studio. Ma, nella radicalizzata polarità tra interno ed esterno, ${ }^{42} \mathrm{le}$ «misure dell'anima» rideterminano anche, complessivamente, il modo in cui l'io percepisce e si rappresenta la propria presenza nella realtà.

Sulla cima della montagna un'ispirazione — sacralmente- improvvisa e immotivata spinge Petrarca a interrogare a caso il piccolo esemplare delle Confessioni che ha sempre tra le mani. E il caso («Forte autem decimus illius operis liber oblatus est» [27]) risponde con parole che miracolosamente, come vuole il rito delle sortes biblicae, illuminano la sua coscienza. Le parole "si offrono" con la tonalità oracolare della rivelazione, sono come il soffio divino della verità, il Verbo. La frase di Agostino mette in comunicazione l'eterno con il tempo: l'assoluto si manifesta tra le pieghe del tempo terreno. E' il miracolo di Dio che fa sentire la sua voce a una creatura sorprendendola nella casualità dell'esistere. Si riaffaccia qui, insomma, la verticalità del rapporto fra uomo e Dio, nella cui metafisica vertigine la cultura cristiano-medievale vede integralmente risolversi il significato della vita. Ciascuno sa di trovarsi in ogni momento sotto lo sguardo di Dio ed è sempre pronto a ricevere e decifrare i segni misteriosi della sua provvidenza. Così: «Nec opinari poteram id fortuito contigisse, sed quicquid ibi legeram, michi et non alteri dictum rebar» (30). Petrarca comprende che la coincidenza che lo ha sconcertato — «obstupui»— non può essere casuale, ma nasconde il cenno che Dio rivolge, precisamente, "a me e non a un altro".

40. Così, ad esempio, «astrologi celum lustrant, astra connumerant [...]; quid imperiis atque urbibus eventurum sit tam audacter tanto ante denuntiant, quid sibi quotidie eveniat non attendunt; lune solisque defectus provident, presentem eclipsim anime non videntes» (F. PETRARCA, Le Familiari, vol. III, cit., p. 212-3).

41. Ivi, p. 212.

42. Un cui rilevantissimo risvolto è la stessa scissione tra libertà incondizionata della coscienza ("Animo quidem sub nullo sum, nisi sub Illo qui michi animum dedit») e accettazione dispregiativamente passiva delle forme del potere («Pars autem mei altera hec terrestris terrarum dominis quorum loca incolit subdita sit oportet») che qualifica, nell'ambito dell'ideologia politica, la figura moderna dell'intellettuale Petrarca. Ed è superfluo dire quanto questa scissione, che abbiamo riassunto con le parole della Invectiva contra quendam magni status hominem sed nullius scientie aut virtutis (in F. PETRARCA, Prose, cit., p. 700), e in termini analoghi si ripresenta nella celebre senile VI 2 a Boccaccio, fosse estranea all'orizzonte mentale di Dante, che non avrebbe mai assimilato alla materiale esteriorità del corpo — in chiave svalutativa — la partecipazione alla vita civile. 
Le parole che egli ha letto, però, sono di Agostino; il quale racconta nelle Confessioni (VIII, 12, 28) che, ugualmente aprendo a caso la Lettera di Paolo ai Romani, ne aveva tratto la scintilla della conversione, proprio come, ancor prima, Antonio era stato fulmineamente convertito da un passo del Vangelo di Matteo ascoltato casualmente. Ed è allora ripensando a quanto era avvenuto ad Agostino, il quale sua volta, in quella circostanza, aveva ripensato a quanto era avvenuto ad Antonio, che lo scrittore può dare alla propria attuale esperienza la pienezza di senso di un messaggio divino. Rileggiamo il brano per intero:

Nec opinari poteram id fortuito contigisse, sed quicquid ibi legeram, michi et non alteri dictum rebar; recolens quod idem de se ipso suspicatus olim esset Augustinus, quando in lectione codicis Apostolici, ut ipse refert, primum sibi illud occurrit: «Non in comessationibus et ebrietatibus, non in cubilibus et impudicitiis, non in contentione et emulatione; sed induite Dominum Iesum Cristum, et carnis providentiam ne feceritis in concupiscentiis vestris». Quod iam ante Antonio acciderat, quando audito Evangelio ubi scriptum est: «Si vis perfectus esse, vade et vende omnia tua quecunque habes et da pauperibus, et veni et sequere me et habebis thesaurum in celis», veluti propter se hec esset scriptura recitata, ut scriptor rerum eius Athanasius ait, ad se dominicum traxit imperium. Et sicut Antonius, his auditis, aliud non quesivit, et sicut Augustinus, his lectis, ulterius non processit, sic et michi in paucis verbis que premisi, totius lectionis terminus fuit... (30-2).

Già Agostino aveva avvertito nella cantilena infantile del «Tolle lege» il "comando divino" ricordandosi di Antonio ${ }^{43}$. Anche per Petrarca il grande tema agostiniano della memoria — «recolens»— svolge il ruolo principale. ${ }^{44}$ La necessità dell'evento, la sua verità e leggibilità, è sancita dal ricordo degli esempi passati, deriva ed è insieme autenticata da una catena di esperienze umane, ha il fondamento in una genealogia di pensieri e di scritture, di pensieri (ipotesi, supposizioni, costruzioni ermeneutiche: «suspicatus esset», «veluti») depositati nelle scritture. Il linguaggio di Dio richiede, per essere inteso, la mediazione delle interpretazioni umane. La trasparenza con cui l'ordine profondo del creato si dà al soggetto emerge solo dai discorsi che ne tramandano e ne rinnovano la scoperta. Il mistero del sortilegium perde così la sua

43. "Et ecce audio vocem de vicina domo cum cantu dicentis et crebro repetentis quasi pueri an puellae, nescio: "Tolle lege, tolle lege". Statimque mutato vultu intentissimus cogitare coepi, utrumnam solerent pueri in aliquo genere ludendi cantitare tale aliquid, nec occurrebat omnino audisse me uspiam repressoque impetu lacrimarum surrexi nihil aliud interpretans divinitus mihi iuberi, nisi ut aperirem codicem et legerem quod primum caput invenissem. Audieram enim de Antonio, quod ex evangelica lectione, cui forte supervenerat admonitus fuerit, tamquam sibi diceretur quod legebatur...» (AGOSTINO, Confessioni (a cura di R. De Monticelli), Milano: Garzanti, 1990, p. 290-2).

44. "Proprio secondo lo schema creato da Agostino, la memoria prende il sopravvento sulla curiositas, l'interiorità sull'estasi davanti al mondo, la preoccupazione della salvezza sull'affezione teoretica, ma anche il riferimento temporale sul rapporto spaziale» (H. BLUMENBERG, La legittimità, cit., p. 368). 
aura sacrale, magica per colorarsi di storicità, ricondotto com'è alla memoria degli uomini, alla storia delle loro interpretazioni. Veramente, «a locis ad tempora». Dalla verticalità dell'immobile architettura simbolica ${ }^{45}$ del cosmo, organizzata secondo la diade alto/basso, Creatore/creatura, all'orizzontalità del tempo della storia.

Se l'enigma del caso si scioglie nel passaggio dall'unicità alla ripetizione e la verità è inscritta nella sequenza dei "sicut", vuol dire che il presente si integra con il passato, il significato del presente si chiarisce alla luce del passato e dei suoi modelli, nella temporalità di un pensiero che si muove - confrontando, collegando - lungo l'arco della storia. Petrarca — si sa - ha largamente coltivato il genere dell'exemplum, dai Rerum memorandarum al De remediis; e di exempla è costellato anche l'epistolario. Nella "consolatoria super quibusdam vite difficultatibus» al frate Giovanni Colonna (Fam. VI 3) l'iperbolico accumulo di esempi rischia di prevaricare il calibro morale dell'argomento sottomettendolo al compiacimento dello storiografo ( Sed quid ago? brevis consolatio in longam historiam versa est») ${ }^{46}$ Addirittura la lettera successiva, la quarta del VI libro, confermando una volta di più l'acutezza di uno scrittore sempre infallibilmente consapevole delle sue scelte («Exemplis abundo, sed illustribus, sed veris, et quibus, nisi fallor, cum delectatione insit autoritas»), ${ }^{47}$ è dedicata proprio, con l'oltranza arguta di una tematizzazione al quadrato, alla dimostrazione, naturalmente per mezzo di esempi, dell'importanza degli esempi: «Quid exempla valeant exemplis ostenditur» è il suo titolo. Gli esempi -la "suppellettile degli esempi» ${ }^{48}$ di cui l'autore ama servirsi con larghezza- legittimano, autorizzano l' "esperienza» individuale, strappandola alla sua opacità, portandola a riconoscersi:

Si ratio queritur cur exemplis interdum affluam, curioseque in his videar immorari, dicam: puto lectorem eo animo esse quo sum ego. Me quidem nichil est quod moveat quantum exempla clarorum hominum. Iuvat enim assurgere, iuvat animum experiri an quicquam solidi habeat, an generosi aliquid atque adversus fortunam indomiti et infracti, an sibi de se ipse mentitus sit. Id sane, preter experientiam que certissima magistra rerum est, nullo melius modo fit, quam si eum his quibus simillimus esse cupit, admoveam. Itaque, sicut omnibus quos lego, gratiam habeo, si michi sepe propositis exemplis hanc experiendi facultatem dederint, sic michi gratiam habituros spero qui me legent ${ }^{49}$.

45. Il «simbolismo che, sistematizzato in metodo di ricerca e di spiegazione», come dice Le Goff, «fa svanire tutta la realtà concreta del tempo della storia» (J. LE GofF, Tempo della Chiesa, cit., p. 10).

46. F. Petrarca, Le Familiari, cit., vol. II, Firenze: Sansoni, 1934, p. 67.

47. Ivi, p. 77.

48. Nel Secretum Francesco riconosce: «Est autem, nisi fallor, grande solatium tam claris septum esse comitibus; itaque fateor talium exemplorum, velut quotidiane supellectilis, usum non reicio»; e Agostino non disapprova: «Aperte equidem nec supellex hec exemplorum displicet...» (F. Petrarca, Secretum, in Prose, cit., p. 178-80).

49. F. PetrarCA, Le Familiari, vol. II, cit., p. 78. 
Nella valutazione dell'umanista, che ne è insieme fruitore in proprio e produttore per le generazioni a venire, gli exempla, smesso il tenore di edificazione religiosa che li caratterizzava nell'uso medievale, danno all'animo l'occasione di sperimentare («experiri») la propria verità («an sibi de se ipse mentitus sit») attraverso l'emulazione fortificante dei «clari homines». Gli esempi generano valori, dischiudono nuove possibilità, innescano e sospingono il movimento della storia:

Omnes qui ante Marium a medicis secabantur, vinciri mos fuerat; quia enim dolorem corporis animi robore superari non posse persuasum erat, vinculorum auxilio utebantur. Primus Marius solutus sectus est, sed post eum pluri$\mathrm{mi}$; cur, queso, nisi quia exemplum viri constantissimi atque fortissimi ad imitandum animos erexit, et ut compatriote sui verbo utar, valuit autoritas? ${ }^{50}$

L'autoritas produce storia: gli esempi forzano le consuetudini, forniscono di volta in volta la misura - aperta, dunque, mobile - dell'umano, tracciano la frontiera, continuamente modificabile, delle sue possibilità. ${ }^{51}$ Il dialogo verticale con Dio, nell'alternativa immutabile della salvezza o della dannazione, non esaurisce più il senso dell'esistenza. Sconvolgendo le coordinate spazio-temporali della cultura medievale, Petrarca introduce decisamente nella visione della realtà la dimensione della storia: è la tradizione dell'umano, custodita nelle parole della letteratura, la garanzia della verità degli uomini. Le scelte, le direzioni dell'agire, la responsabilità della condotta sono svincolate dalla relazione esclusiva con la fede, con la presenza atemporale del divino, e ritrovano il loro riferimento nei valori espressi dagli esempi storici. In chiusura la familiare VI 4 rievoca la conversione di Agostino, ovvero l'esempio di Agostino che imita un esempio:

Unum etiam nunc exemplum tibi notissimum quominus interseram, temperare nequeo. Siquidem, quem vite callem arriperet diutissime fluctuanti Augustino, et Antonii Egiptii et Victorini rethoris ac martyris profuit exemplum,

\section{Ibidem., p. 79.}

51. Identificando nelle Familiares di Petrarca l'avvento, per la prima volta nella cultura occidentale, dell'autenticità dell'Io (laddove in Agostino «l'autobiografia era soltanto una teologia in prima persona»), Paul Veyne ha scritto: "Con la sua testimonianza, Petrarca mostra come un individuo, che altri non è se non lui stesso, abbia riscoperto e interiorizzato la lezione di S. Agostino. Né tipo né esempio, ma uomo tra gli uomini; S. Agostino incarnava una lezione; Petrarca racconta di essere arrivato a imparare questa lezione. La sincerità consiste nel testimoniare che una cosa è umanamente possibile, dal momento che un uomo qualsiasi l'ha fatta o subita; ogni parola veridica è un seme che può far germogliare delle idee in altri uomini. La Verità non è più insegnata in forma di lezioni: ogni uomo può scoprire la propria ascoltando la storia di un altro uomo, ingenuo quanto lui. All'antico dogmatismo ha fatto seguito una pedagogia di tutti ad opera di tutti. Ognuno può riscoprire a sua volta l'America, dal momento che io l'ho potuto fare. E' interessante per tutti che una certa cosa sia capitata a me, uomo qualsiasi; potrà dunque capitare a chiunque altro» (Paul VeYne, La poesia, l'amore, l'occidente (L'elegia erotica romana), Bologna: Il Mulino, 1985, p. 280-1). 
nec non et illorum duorum in rebus agentium apud Treveros repentina conversio; quam cum sibi Pontianus miles imperatorius enarrasset -ipsius Augustini verba tenes, octavo, nisi me memoria frustratur, Confessionum libro posita—, «Exarsi» inquit, «ad imitandum; ad hoc enim et ille narraverat». ${ }^{52}$

L'epistola del Ventoso, capitolo culminante della petrarchesca imitatio Augustini, ${ }^{53}$ celebra questa funzione — più che etica - conoscitiva, epistemologica dell'imitazione. In essa l'"esempio» come schema di interpretazione della realtà è il principio formale del testo, che ne impronta e orchestra capillarmente la composizione, pervadendone le fibre nel segno intensivo della ripetizione, del confronto, del raddoppiamento.

E' un passo capitatogli fortuitamente sotto gli occhi mentre rileggeva Livio a indurre Petrarca a compiere l'ascensione del Ventoso: «Relegenti pridie res romanas apud Livium forte ille michi locus occurrerat, ubi Philippus Macedonum rex - is qui cum populo Romano bellum gessit- Hemum montem thesalicum conscendit» (2). Prima di impersonare la parte di Agostino, e di Antonio, nella scena cruciale della conversione, il narratore-protagonista, dun-

52. F. Petrarca, Le Familiari, vol. II, cit., p. 80.

53. Un'imitazione che, naturalmente, contempla l'infedeltà nei particolari: sui punti in cui il ricalco che ne fa Petrarca si diversifica dalla conversione di Agostino com’è descritta nelle Confessioni v. M. GuglielminetTI, Memoria e scrittura (L'autobiografia da Dante a Cellini), Torino: Einaudi, 1977, p. 109-10. Soprattutto, mentre per Antonio e Agostino «l'evento si conclude con una repentina, folgorante conversione, [...] nel caso del Petrarca, la scena serve a mettere in luce solo la necessità dell'autoesame, [...] lasciando però del tutto impregiudicato il cambiamento della propria vita», esaurendosi, insomma, in "una mera postulazione di carattere gnoseologico» (B. MARTINELLI, op. cit., p. 30-1). L'aspetto della differenza è accentuato da R.M. DuRLiNG (op. cit., p. 318-9): «E” evidente che il Petrarca ha sempre in mente la vicenda milanese e che al centro della sua concezione si pone il rapporto con $i$ modelli. Ma qui si tratta non della riuscita definitiva, bensì del contrario. Ciò che domina è il senso della mancanza, della differenza. [...] Sulla vetta Francesco è in grado di misurare la distanza che lo separa dai modelli. Quando l'oracolo distrugge le sue pretese, suggerendo che avrebbe fatto meglio a rimanere a casa, è come se il testo lo avesse circondato, insidiato proprio nel momento in cui non lo aspettava. [...] Così proprio il parallelismo più importante tra lui e il modello, l'esperienza dell'oracolo, si rivela negativo, ironico». Le implicazioni "filosofiche» del "contrasto fra Agostino e Petrarca» sono invece così enucleate da P. VEYNE (op. cit., p. 293): "Agostino racconta "oggettivamente" che aprendo a caso il libro dell'apostolo vi lesse l'ordine divino di rompere col peccato; il suo caso testimonia che Dio può inviare un messaggio a un uomo per questo tramite. Petrarca testimonia, per parte sua, che un uomo ha il diritto di supporre che Dio gli invii un messaggio per questo tramite. Agostino racconta un fatto esemplare, Petrarca dà un esempio di audacia personale. Agostino non ha un attimo di dubbio o piuttosto di esitazione sul senso e sulla destinazione del messaggio; non dice una parola circa i sentimenti da lui provati: sono sottintesi. Petrarca si limita a dire che non ha potuto fare a meno di credere che il messaggio era destinato a lui, senza pronunciarsi esplicitamente sulla realtà oggettiva di questa specie di miracolo. Per S. Agostino il miracolo perderebbe ogni valore, se fosse stato una pura illusione soggettiva; per Petrarca, resta il fatto che tale illusione soggettiva ha una realtà, quella di essere stata creduta. Per Agostino l'elemento soggettivo trova un limite nella verità che traspare; Petrarca considera invece i suoi sentimenti soggettivi come esempio di una verità possibile». All'assolutezza metafisica sta subentrando un nuovo statuto, soggettivo e storico, della verità. 
que, ha un altro "doppio" in Filippo, ${ }^{54}$ così come il monte Emo corrisponde al Ventoso e, soprattutto, la lettura a caso della storia romana anticipa e prepara, quasi un presagio, quella delle Confessioni: «forte». Un libro accende la curiosità di ammirare la natura, un altro libro ammonisce all'introspezione: è la costellazione delle parole che ispira i giudizi e le risoluzioni della vita ${ }^{55}$. Ma questo ordito di ripetizioni e differenze si dissemina addirittura, in scala più sottile, nelle cellule motiviche e nei coaguli espressivi della scrittura: lo stupore dell'altitudine, come abbiamo già constatato, che si prolunga in quello causato dal «verbum» agostiniano, oppure il dato fisico "nubes erant sub pedibus» (17) a cui fa eco la metaforizzazione di "ut elatos terrenis impulsibus appetitus sub pedibus haberemus» (34). Con una trama di consonanze verbali, di rispondenze strutturali e simmetrie narrative (situazioni replicate, gesti che ritornano), in cui si sarebbe anche tentati di vedere una sorta di secolarizzazione o tecnicizzazione della "figura» auerbachiana, l'autore impone alla precarietà delle cose e dei fatti la misura coerente di una storia di rapporti, costruendo, elaborando compositivamente un significato delle azioni umane che non ha, perciò, il crisma teologico della necessità, non è più impresso nella mappa simbolica dell'universo come segno dell'ordine di Dio.

La verità e l'ordine del mondo coincidono, alla fine, con la verità e l'ordine della rappresentazione letteraria. ${ }^{56} \mathrm{E}$ l'ascesi autentica sarà quella dello stile, la cui emblematica, «trascendentale» (Contini), classica stabilità Petrarca destina, unica «episteme» possibile, a dominare la fuga del tempo.

54. Si noti la correlazione che intreccia affinità e disuguaglianza: «Ceterum, ut illo omisso, ad hunc montem veniam, excusabile visum est in iuvene privato quod in rege sene non carpitur» (3). E - ulteriore corrispondenza - il pastore che, unico, ha preceduto i due fratelli nel tentativo era stato mosso dallo stesso ardore giovanile («dicens se ante annos quinquaginta eodem iuvenilis ardoris impetu supremum in verticem ascendisse» [7]; dove si riverbera perfino il «Cepit impetus...» del par. 2).

55. E infatti, se «l'ottica dello sguardo [...] domina tutta l'epistola del Ventoso", "anche l'attività del legere, come nelle Confessioni, si rivela egualmente capitale, e nella progettazione $\mathrm{e}$ gestione della vicenda, e nell'elaborazione del testo" (B. MARTinelli, op. cit., p. 42). E' questo, d'altronde, nella "grande alternativa» di "contemplazione e leggibilità, natura e libro», «il prototipo creato da Petrarca, il quale ha mirato con fermissima intenzione alla visione della natura e tuttavia porta con sé il libro; neppure la spettacolare visione della natura è capace di trattenerlo dal leggervi» (H. BLUMENBERG, La leggibilità del mondo, Bologna: Il Mulino, 1984, rispettivamente p. 322 e 291).

56. "Alla mutatio, congenita a qualsiasi manifestazione della natura - a questa terribile forza, cui altrimenti non si può resistere-, viene contrapposto [da Petrarca e Boccaccio] chiaramente un ordo. Ma questo ordine [...] è e può essere solamente estetico, dunque, in senso lato, linguistico» (A. AsOR ROSA, "La fondazione del laico», in A. AsOR RosA (a cura di), Letteratura italiana. 5. Le Questioni, Torino: Einaudi, 1986, p. 117). D'altra parte, nella familiare del Ventoso "l'allegoresi è identificata come atto linguistico», mentre "il mondo dei segni si sta separando da quello dei referenti, i segni rischiano di diventare solamente segni» (R.M. DURLING, op. cit., p. 306 e 323). 